\title{
Towards the alternativization of health? a commentary on Joana Almeida's notion of camisation
}

\section{Commentary}

In July 2018, a one-day plenary session on "Sociology, theory and Complementary and Alternative Medicine" at XIX ISA World Congress of Sociology, brought together leading scholars to set the agenda for the field. The congress highlighted sociological disregard for theorization of traditional medicines ${ }^{1}$ (TMs) and complementary and alternative medicines (CAMs), despite avalanche of studies in the last 15-20years. The sociological understanding of TM and CAM require an analytical and conceptual framework that can be applied across contexts and variations. Without doubt, conceptual and theoretical frameworks espousing the current state knowledge on CAM and TM have generally been limited. However, recent attempt by Joana Almeida at proposing camisation, as a conceptual/theoretical framework for the analysis of CAM in western society is both timely and welcome. ${ }^{1}$

Camisation was coined by Almeida in 2012 in her doctoral dissertation that explored the relationship between CAM, the medical profession and the State regulation in Portugal, using acupuncture and homeopathy practitioners, as useful cases. The notion of camisation was further refined following its presentation at XVIII ISA World Congress of Sociology in Japan (2014) and international conference on Traditional, Complementary and Alternative Medicine in Poland (2016) as resolving everyday human problems through CAM framework. Camisation is defined as 'a process through which everyday human problems are transformed into health problems which are treated in CAM terms and within a CAM framework' (Almeida 2012: 92). Almeida's thesis is premised on the integration and institutionalisation of CAM into orthodox western medicine through the framework of camisation. Central to this idea is the argument that camisation can contribute to re-medicalisation (the process whereby the medical meanings of a disease are restored) or de-medicalisation (the process whereby human problems come to be redefined in nonmedical terms).

Almeida's notion of camisation, however, represents a promising step towards developing a useful sociological understanding of CAM and TM in contemporary global society. Despite this important contribution, the concept of camisation is problematic for two reasons that I seek to articulate briefly here.

First, the fundamental premise on which camisation is conceptualised is faulty. Camisation stems from CAM, and CAM is still a controversial concept in Sociology that has not been widely accepted. Almeida (2012: 19) acknowledges the controversies trailing the terminology of CAM:

The terms "complementary" and "alternative" run parallel to each other despite suggesting different relationship with biomedicine. The term "complementary medicine" refers to the use of CAM alongside conventional medicine, such as using acupuncture in addition to

${ }^{1}$ Traditional Medicine is regarded as Alternative Medicine (AM) outside their traditional context or jurisdiction.
Volume 12 Issue I - 2019

\author{
Ayodeji B Ogunrotifa \\ Royal Holloway, University of London, UK
}

Correspondence: Ayodeji B Ogunrotifa, Centre for Criminology and Sociology, Royal Holloway, University of London, Egham, Surrey TWO OEX, UK, Email Ayodeji.Ogunrotifa.2008@live.rhul.ac.uk

Received: January 07, 2019 | Published: February 12, 2019

other orthodox medical procedures like conventionally prescribed medicines to help reduce or manage pain.

Following Holliday, ${ }^{2}$ and $\mathrm{Gale}^{3}$ further deepened the debate around the controversies associated with CAM, by positing that 'alternative medicine' and 'complementary medicine' are fundamentally two medical systems that are different in practice. Alternative medicine is widely accepted in developing countries because its knowledge, practise and skills evolved out of indigenous and traditional ways of dealing with health and illness. Whereas in western countries, alternative medicine does not have appeal because of the biomedical dominance that has framed the language of health and healing, and thus, foregrounds the dominance of orthodox/modern medicine.

Nevertheless, both alternative and orthodox medicine is fundamentally different within the context of the contrasting ontologies and epistemologies that underpinned their practice and development. Alternative medicine (or TM) does not need orthodox medicine to perform its functions, and therefore, do not seeks to collaborate with it. What necessitated the emergence of CAM as a terminology was the growing crisis in the ontology and epistemology of scientific medicine that necessitated the accommodation of alternative medicine into orthodox medicine. This crises in the ontology and epistemology of orthodox medicine are manifested in 'changed pattern of morbidity evidenced by the prevalence of chronic against acute conditions in the industrialised societies in which medicine gradually began to realise the inadequacy of its theoretical and material resources'. ${ }^{4}$

The adventure into alternative medicine was one of the strategies utilised by biomedicine/orthodox medicine to mitigate the crisis or inadequacies of its ontology and epistemology, and therefore recognised the role that alternative medicine can play in treatment of illness, particularly in cases where disease entities are either intractable or difficult to identify or where there appears to be no obvious physiological basis for compliant.

Alternative medicine was therefore utilised to complement orthodox medicine in solving the problems of its epistemology and ontology. The aspect of alternative medicine that was appropriated into orthodox medicine are regarded as 'complementary medicine', while other forms of AM that are considered incompatible with orthodox medicine were neglected and regarded as fringe. The notion 
of 'complementary medicine' was later redefined as 'Complementary and Alternative medicine' by British Medical Association (BMA) in $1993 .^{3}$

Nevertheless, both alternative medicine and orthodox medicine are different and run parallel to each other due to contrasting ontologies and epistemologies. Therefore, the framing of CAM by my own reckoning is best defective, inoperative and nugatory. As a corollary to this popular saying that "you cannot build something on nothing", Almeida's notion of camisation cannot be built on defective CAM. CAM should therefore be deconstructed as a sociological concept and thrown into rubbish heap of intellectual history, while AM should constitute a new frame of conceptual understanding. In this sense, acupuncture, homeopathy and others that are currently coalescence into CAM, should be classified as AM because the ontology and epistemology underpinning these forms of AM are incompatible with orthodox medicine, and therefore, there is no basis for complementarity.

The complementary aspects of alternative medicine that was incorporated into orthodox medicine are of small-scale and occurred within the limit in which ontology and epistemology of orthodox medicine can permit (for examples, acupuncture and homeopathy). Instead of CAM to be struggling to achieve 'professional status especially in the form of statutory regulation's in the name of complementarity and integration, alternative medicine and orthodox medicine should rather go their separate ways because they are different and unrelated. This separation would afford AM the opportunity to develop its own practice, methods and technique, and achieve standardization and professionalization like that of orthodox medicine.

The proponents and defenders of orthodox medicine should acknowledge the limit of orthodox medicine and recognise the opportunities that AM offers in providing alternative (healthcare) to the shortcomings of orthodox medicine. Therefore, AM practitioners should be accorded due recognition that it deserves, and be standardised and autonomous like orthodox medicine, to operate within the institutionalisation of healthcare. This is because orthodox medicine cannot resolve most of the human/health problems, and therefore AM must be accorded the respect to resolve human problems that are within its competence, but outside the jurisdiction of orthodox medicine. Therefore, camisation is flawed because the terminology of CAM that underpins its conceptualisation is defective.

Secondly, camisation is purely western conception that fails to take social and cultural context of health and healing in developing countries into consideration. Almeida is only interested in the form of alternative medicine like acupuncture, and homeopathy (or what was regarded as CAM) that can be incorporated into orthodox medicine. Thus, obscuring other forms of alternative treatments that are useful and contributing to the health and illness in developing countries, but are considered as fringe and unconventional within the framework of orthodox medicine. There are other forms of alternative treatments that are neglected and marginalised, given the biomedical dominance of orthodox medicine. For instance, Sams ${ }^{6}$ observed the use of Kohl (a Hausa traditional medicine) for the treatment of eyes problem in Niger. Other studies on AM includes the use of shamanism for the treatment of depression and mental health in China ${ }^{7-9}$ and the use of indigenous spiritism for the treatment of alcoholism in Puerto Rico. ${ }^{10}$

What will then happen to kohl, shamanism, spiritism and other forms of alternative treatment that would never be accepted or incorporated into orthodox medicine due to contrasting ontologies and epistemologies? Therefore, the concept of camisation is limited and cannot be applied globally or in different context outside western countries. The lay understanding of kohl, shamanism and spiritism to their users is that of alternative medicine on its own right, that do not complement orthodox medicine in any way.

\section{Moving forward? the alternativization of healthcare}

Having critiqued CAM, as well as camisation as a useful analytical concept, this paper proposes 'alternativization', a concept that offers a new analytical frontier, capable of explaining wider coverage of alternative medicine use in different contexts. However, alternativization was coined by Chris Macdonald in 2012 to articulate extensive use of alternative healthcare as a response to the problem posed by medicalisation. Alternativization was therefore, defined as 'the tendency to understand normal events (for example, menstruation, pregnancy, childbirth, menopause) as pathological states requiring intervention by practitioners of alternative therapies. ${ }^{5}$ Macdonald argued that the growing use of alternative therapies, constitute risk to women's health, amidst the depreciation of its scientific value. Macdonald therefore argues that women who avoid the negative aspects of medicalization by using alternative therapies are moving from "frying pan" to "fire". Therefore, alternativization was purposively coined to critique and lash out at women and others who dare to use alternative medicine.

However, like other defenders of mainstream orthodox medicine, Macdonald is yet to fully accept the inadequacy and limitation of orthodox medicine in resolving the problems of health and healing or why alternative therapy users are disenchanted with modern orthodox medicine, a development that has proved to be real in both western and developing countries contexts. Also, Macdonald's definition of alternativization is limited in capturing the social and cultural understanding of alternative therapies in developing countries and why everyday human problems like depression, seasonal affective disorder, insomnia, pre-menstrual syndrome, stress, menstruation, menopause and infertility are regarded as health problems requiring alternative treatment. Like Almeida, Macdonald is examining the usefulness of alternative therapies within the lens of western orthodox medicine, and from the standpoint of the western context, and as such, his definition and understanding of alternativization is thus limited and one-sided. Given these shortcomings associated with Macdonald's idea of alternativization, this paper therefore proposes an encompassing definition of alternativization.

As a corollary to camisation, alternativization is the process through which everyday human problems are considered as health problems requiring traditional/alternative medical solutions and are such treated within the framework of traditional/alternative medicine. Unlike Almeida's proposition that vacillate between whether camisation can foster re-medicalisation or de-medicalisation, alternativization rejects both medicalisation and pharmaceutisation. Central to this idea of alternativization is the recognition of AM as alternate and autonomous form of medicine that does not require any link (divergence) with orthodox medicine. Alternativization as a sociological concept is more encompassing than camisation because its remit of engagement is more than western context. The explanatory power of alternativization will be suited in exploring the use of $\mathrm{AM}$ in both developing countries and western contexts. Therefore, alternativization as a sociological concept seems more global in its scope of engagement than camisation. 
To conclude, what I am proposing (alternativization) is a rejection of Almeida's notion of camisation. It is my candid hope that these debates continue in intellectual and constructive spirit in which they are intended, and in doing so, we move to a useful and richer analytical framework.

\section{Acknowledgments}

None.

\section{Conflicts of interest}

Author declares there is no conflict of interest.

\section{References}

1. Almeida J. Towards the camisation of health? The countervailing power of CAM in relation to the Portuguese mainstream healthcare system. $\mathrm{PhD}$ thesis, Royal Holloway, UK: University of London; 2012.

2. Holliday KV. Folk or Traditional Versus Complementary and Alternative Medicine: Constructing Latino/a health and illness through biomedical labelling. Latino Studies. 2008;6(4):398-417.
3. Gale N. The Sociology of Traditional, Complementary and Alternative Medicine. Sociology Compass. 2014;8(6):805-822.

4. Igun UA. Medical Sociology: An Introduction. Ibadan: Shaneson CI. Press; 1988.

5. Welsh S, Kelner M, Wellman B, et al. Moving forward? Complementary and alternative practitioners seeking self-regulation. Sociology of Health \& Illness. 2004;26(2):216-241.

6. Sams K. Engaging conceptions of identity in a context of medical pluralism: explaining treatment choices for everyday illness in Niger. Sociology of Health \& Illness. 2017;39(7):1100-1116.

7. Winkelman M. Shamans, priests, and witches: A cross-cultural study of magico-religious practitioners. Tempe: Anthropological Research Papers. USA: Arizona State University; 1992.

8. Winkelman M. Shamanism: A natural ecology of consciousness and healing. London: Bergin and Garvey; 2002.

9. Winkelman M. Shamanism as the original neurotheology. Zygon. 2004;39(1):193-217. 